\title{
Direct regulation of rhodopsin 1 by Pax-6/eyeless in Drosophila: evidence for a conserved function in photoreceptors
}

\author{
Guojun Sheng, ${ }^{1}$ Eric Thouvenot, ${ }^{1}$ Dietmar Schmucker, ${ }^{2}$ David S. Wilson, ${ }^{1}$ and Claude Desplan ${ }^{1,3}$ \\ ${ }^{1}$ Laboratory of Molecular Genetics, Howard Hughes Medical Institute, and ${ }^{2}$ Laboratory of Developmental Neurogenetics, \\ The Rockefeller University, New York, New York 10021 USA
}

Pax-6 is a transcription factor containing both a homeodomain (HD) and a Paired domain (PD). It functions as an essential regulator of eye development in both Drosophila and vertebrates, suggesting an evolutionarily conserved origin for different types of metazoan eyes. Classical morphological and phylogenetic studies, however, have concluded that metazoan eyes have evolved many times independently. These apparently contradictory findings may be reconciled if the evolutionarily ancient role of $\mathrm{Pax}-6$ was to regulate structural genes (e.g., rhodopsin) in primitive photoreceptors, and only later did it expand its function to regulate the morphogenesis of divergent and complex eye structures. In support of this, we present evidence that eyeless (ey), which encodes the Drosophila homolog of Pax-6, directly regulates rhodopsin 1 (rh1) expression in the photoreceptor cells. We detect ey expression in both larval and adult terminally differentiated photoreceptor cells. We show that the HD of Ey binds to a palindromic HD binding site P3/RCS1 in the rh1 promoter, which is essential for $r h 1$ expression. We further demonstrate that, in vivo, P3/RCS1 can be replaced by binding sites specific for the PD of Ey. P3/RCS1 is conserved in the promoters of all Drosophila rhodopsin genes as well as in many opsin genes in vertebrates. Mutimerized P3 sites in front of a basal promoter are able to drive the expression of a reporter gene in all photoreceptors. These results suggest that Pax-6/Ey directly regulates rhodopsin 1 gene expression by binding to the conserved P3/RCS1 element in the promoter.

[Key Words: Pax-6; eyeless; rhodopsin; eye evolution; photoreceptor cell; homeodomain]

Received February 18, 1997; revised version accepted March 25, 1997.

In 1977, Salvini-Plawen and Mayr proposed, after careful anatomical and phylogenetical analyses of various types of photoreceptive organs, that metazoan eyes have originated independently in more than 40 different phyletic groups (Salvini-Plawen and Mayr 1977). Recent molecular genetic studies on eye development in both vertebrates (Hill et al. 1991; Ton et al. 1991; Oliver et al. 1995) and Drosophila /Cheyette et al. 1994; Quiring et al. 1994; Halder et al. 1995a) showed, however, that several key regulatory genes for eye organogenesis (e.g., Pax-6, sine oculis) are conserved from Drosophila to vertebrates. Mutations in the Pax-6 gene in vertebrates and its Drosophila homolog eyeless (ey) lead to reduced or absent eye structures (for review, see Gruss and Walther 1992; Strachan and Read 1994). Ectopic expression of Drosophila ey or vertebrate Pax-6 in Drosophila larval imaginal discs leads to transformation of leg, wing, and antennal structures into morphologically normal eyes (Halder et al. 1995a). These studies led to the hypothesis

${ }^{3}$ Corresponding author.

E-MAIL desplan@rockvax.rockefeller.edu; FAX (212) 327-8370. that $P a x-6$ is the master regulatory gene of eye development, at least in Drosophila, and possibly throughout Metazoa (Halder et al. 1995a). A model of eye evolution that reconciles the classical morphological studies and these recent molecular findings suggests that, in a primitive metazoan organism, Pax- 6 would have directly controlled genes involved in photoreception (Zuker 1994; Halder et al. 1995b). During the later evolution of independent metazoan lines, Pax-6 would have taken on an increasingly complex role in regulating genes responsible not only for photoreception but also for anatomically complex structures for light gathering and image formation, such as those of insects and vertebrate eyes. A prediction of this model would be that Pax- 6 may still, in the context of highly evolved eyes, maintain its original regulatory role of activating the structural genes required for light detection.

Pax-6 gene is a member of the Pax gene family, which encodes two conserved DNA-binding domains, a Pairedtype homeodomain (HD) and a Paired domain (PD) (Gruss and Walther 1992; Strachan and Read 1994; Quiring et al. 1994). The DNA-binding properties of these 
two domains have been studied extensively (Czerny et al. 1993; Wilson et al. 1993; Epstein et al. 1994a,b;Chalepakis and Gruss 1995; Czerny and Busslinger 1995); and the crystallographic structure of both domains, in complex with DNA, has been solved (Wilson et al. 1995; $\mathrm{Xu}$ et al. 1995). The Pax-6 HD, like other Paired-type HDs, is capable of cooperative homodimerization upon binding to a specific DNA site called P3-TAATYNRATTA ( $\mathrm{Y}=\mathrm{C}$ or $\mathrm{T} ; \mathrm{R}=\mathrm{G}$ or A) (Wilson et al. 1993; Czerny and Busslinger 1995).

In Drosophila, ey transcripts are first detected at the germ band elongation stage in every segment of the ventral nerve cord, in the brain, and in the primordia of the eye discs. Later in embryogenesis, it becomes confined to the brain and the eye disc primordia. In larval stages, it is expressed throughout the eye discs until the third instar, when ey is detected only in cells anterior to the morphogenetic furrow and possibly a few cells posterior to the furrow (Quiring et al. 1994). The expression patterns of ey in pupa and adult have not been reported.

In this paper we present evidence that Ey directly regulates rhodopsin 1 (rh1) expression in photoreceptor cells through the dimeric binding of its HD to rh1 promoter. We show that $e y$ is expressed at several stages during eye development in both larval and adult terminally differentiated photoreceptor cells when $r h$ genes are expressed. We demonstrate that the HD of Ey binds to the rh1 promoter to an element called P3/RCS1 essential for the expression of $r h 1$ as well as of other rhodopsin genes. We further show that, in vivo, P3/RCS1 can be replaced by binding sites specific for the PD of Ey. When we replace the P3/RCS1 with a sequence containing both the HD- and PD-binding sites, the activity of this promoter is enhanced greatly, strongly suggesting that the transcription factor that binds to the P3/RCS1 sequence is Ey, or another very close homolog of Ey in Drosophila with both a PD and a HD. Multimerized P3 sites in front of a basal promoter are able to drive the expression of the $l a c Z$ reporter gene in all photoreceptors in the compound eye as well as in the ocelli. These results support the hypothesis that the evolutionarily ancient role of Pax-6/ Ey was to regulate structural genes (e.g., $r h$ ) in primitive photoreceptors.

\section{Results}

ey is expressed in adult Drosophila retina

The model that Pax- 6 function evolved from an ancestral role in regulating photoreceptor gene expression (Zuker 1994; Halder et al. 1995b) suggests that Drosophila ey may not be required only at early steps for the initiation of photoreceptor development (Halder et al. 1995a), but also in later stages when terminal photoreceptor-specific genes are expressed. The embryonic and larval expression patterns of $e y$ are consistent with the early requirement of this gene: It is expressed in the eye primordia in the embryo and before the morphogenetic furrow at the time of photoreceptor differentiation in the third instar larva. No report of later expression was available. We tested for the presence of ey transcripts in the pupal and the adult stages when terminal photoreceptor specific genes (e.g., $r h)$ are expressed. First, total RNA from adult heads or bodies was prepared and ey transcripts were quantified using RT-PCR with primers specific for either the ey PD or the ey HD. Transcripts could be detected with both sets of primers in adult heads, whereas only a very weak signal was detectable in adult bodies (Fig. 1A). Both the HD- and the PD-specific amplified fragments were cloned and their sequences matched the published ey sequence (Quiring et al. 1994) (data not shown). To determine whether the expression of ey in adult head is localized to the eyes, we performed quantitative RT-PCR experiments with RNA prepared from heads of either wild-type or eyes absent $\left(e y a^{3}\right)$ flies. ey $a^{3}$ flies have strongly reduced compound eyes that have $-25 \%$ of the wild-type number of ommatidia. RT-PCR with the ey HD- and PD-specific primers showed that the wild-type head RNA has 2.5 times more ey transcript than that of eya flies (Fig. 1B). This suggests that the majority of the ey transcripts detected in the adult head are localized to the compound eyes.

\section{A novel form of ey transcript is expressed in the adult} stage

It has been reported that $e y$ has two distinct transcript forms: an embryonic E10 form, which starts with exon 1 (37 amino acid) and skips exon 2 (56 amino acid), and a larval D1 form, which starts with exon 2 (Quiring et al. 1994). The rest of the transcript, which encodes the PD and HD, is the same for both E10 and D1. The exact $5^{\prime}$ boundary of exon 2 is not well-defined and the larval D1 transcript may use a transcription initiation site within the first intron. We detected a novel form of ey transcript from the adult head cDNA. It contained both the first and the second exons. Exon 2 was in frame with exon 1 and its $5^{\prime}$ boundary was 15 base pairs (encoding DNVIA) in front of the first Met reported in the D1 transcript (Quiring et al. 1994). This suggests that the adult-specific transcript contains 98 amino acids before exon 3, which encodes the Paired domain. Although it has been reported that Pax- 6 gene expression and its functions can be regulated by a variety of mechanisms like alternative promoters, alternative splicing, and post-translational processing (Carriere et al. 1993; Epstein et al. 1994b; Plaza et al. 1995; Zhang and Emmons 1995), it is unclear whether different forms of ey transcript in Drosophila play different roles.

Late ey expression starts in the late pupal stage and coincides with rhodopsin gene expression

Although ey is not expressed posterior to the progressing morphogenetic furrow in the eye disc of third instar larvae (Quiring et al. 1994), we detected ey expression at the adult stage. We reasoned that adult expression of ey may be of importance and may indicate a function of ey that 
B

A

PD Specific
HD Specific

Figure 1. (A) Total RNA was extracted from wild-type fly heads and bodies for RT and PCR. Specific PD and HD bands were detected with the head RNA (lane 1). RNA extracted from the bodies gave only very weak $\mathrm{PD}$ and $\mathrm{HD}$ bands (lane 2). In either the head or the body RNA, no specific band for PD, HD, or RP49 were detectable without reverse transcriptase (lanes 3,4). (B) RT-PCR with wild-type (open bars) and ey $a^{3}$ (hatched bars) head RNA extracts. Quantification of HD and PD bands was normalized with RP49. Relative amount of both the PD and the HD showed $\sim 2.5$ times less mRNA in ey $a^{3}$ head RNA extracts than in wild-type extracts. This indicates that $-80 \%$ of adult head ey transcripts are expressed in the compound eyes and the optic lobes. (C) RT-PCR using a total RNA preparation from late third instar (lane 1), pupal (lanes 2-6), and adult stages (lanes 7,8). ey primers for RT-PCR are in the HD region and do not distinguish different forms of ey transcripts (e.g., E10, D1, and adult). ey transcripts were present in most pupal stages with the lowest levels at $36 \%-48 \%$ pd. $r h 1$, as well as other $r h$ genes, started to be transcribed at $60 \%-72 \%$ pd, whereas a rapid increase took place between $72 \%$ and $84 \%$ pd.

is distinct from that required for the initial phases of eye development. The transition from the patterning of the eye discs to the expression of terminal photoreceptorspecific genes occurs during late pupal development. Thus, we looked at the level of ey transcripts at different stages of pupal life. The movement of the morphogenetic furrow ends at $10 \mathrm{hr}$ after pupariation (AP), which is $\sim 10 \%$ of pupal development (pd). Soon after, the eye disc everts at $12 \mathrm{hr}$ AP (Wolff and Ready 1993). The expression of terminal photoreceptor-specific genes starts after the end of cellular patterning of the eye disc, at $\sim 55 \mathrm{hr}$ $\mathrm{AP}$, when all the cell types in each ommatidium have been generated, and the spatial arrangement of these cells and the elimination of surplus cells are complete. We looked, by RT-PCR, for the presence of ey expression in late third instar larvae, five different pupal stages $10 \%$ $1 \%, 12 \%-24 \%, 36 \%-48 \%, 60 \%-72 \%$, and $84 \%-96 \%$ pd and young adults $(<8 \mathrm{hr}$ after eclosion) (Fig. 1C). We observed an initial decrease of ey expression in the first half of the pupal life $(0 \%-48 \%$ pd) with barely detectable $e y$ transcripts during $36 \%-48 \%$ pd. The ey transcripts started to accumulate again starting at $60 \%-72 \% \mathrm{pd}$, reaching the adult level of expression immediately after eclosion. We were interested to know whether the timing of ey expression in the second half of the pupal life was consistent with its putative role in turning on terminally differentiated genes. We measured the timing of expression of one group of these genes, the rh genes, during pupal development. We used specific primers for rh1 to measure its expression level. Very weak expression of $r h 1$ was detected starting from $60 \%$ to $72 \%$ pd (Fig. 1C); a sharp increase was observed from $72 \%$ to $84 \%$ pd, and close to adult level was seen at $84 \%-96 \%$ pd. This expression pattern is similar to that observed by RNA Northern analysis (Kumar and Ready 1995). By using degenerate primers, we detected an almost identical expression pattern for all $r h$ genes combined (Fig. 1C). These data show that the last wave of ey expression coincides with the rapid accumulation of $r h$ transcripts in the late pupal life.

ey is expressed both early and late in Bolwig organ (larval photoreceptor) cells

Interestingly, we also observed a similar pattern of ey expression during the development of larval visual system, the Bolwig organs (BO). Each BO consists of 12 photoreceptors that develop during the second half of embryogenesis (Bolwig 1946; Green et al. 1993). At the end of a stage 12 embryo, the precursor cells of the BO can be identified by monitoring the expression of the Krüppel $(K r)$ gene (Schmucker et al. 1992, 1997). Expression of $K r$ in $\mathrm{BO}$ precursor cells is visible in four to six cells in the posterior head region of late stage 12 embryos. By stage $13, \mathrm{Kr}$ expression can be detected in all $12 \mathrm{BO}$ precursor cells and is maintained thereafter. Although ey is known 
to be critically involved in the development of adult compound eyes, its function in the development of the BO has not been described. We analyzed whether ey is expressed in the BO precursors. By using double in situ labeling we were able to detect colocalization of the $\mathrm{Kr}$ and ey transcripts in $\mathrm{BO}$ precursor cells at the end of stage 12 (Fig. 2A,B). We found this coexpression in at least two BO precursor cells, but it remains unclear how many cells in total do coexpress ey and $K r$. It is possible that there is a wave of BO cell differentiation, similar to the morphogenetic furrow movement in eye imaginal discs where ey is expressed only in undifferentiated cells anterior to the furrow (Quiring et al. 1994). From embryonic stages 13 to mid-stage 16 we find no expression of ey in the differentiating $\mathrm{BO}$ cells (Fig. 2C). Thus, ey is down-regulated and absent during most phases of $\mathrm{BO}$ development, which include morphogenetic movement and axonal growth, elongation, and projection (Schmucker et al. 1992, 1997). Yet at the end of stage 16, a second phase of ey expression in the BO starts (Fig. 2D). Judging by the size of the $\mathrm{BO}$ cell clusters that express ey at the end of stages 16 and 17, we conclude that ey is expressed in all BO cells. The function of this late ey expression in $\mathrm{BO}$ cells may be to regulate the expression of genes specific for terminally differentiated photoreceptor cells (e.g., $r h$ genes), like in the adult compound eyes. The expression pattern of ey in BO cells is consistent with the pattern observed in adult eyes.

\section{ey $H D$ binds to the P3/RCS1 site in the rh1 promoter}

We then tested whether ey expression in late pupal and adult stages is responsible for regulating terminal photoreceptor-specific genes. Several such genes like $r h$ genes are conserved throughout evolution. It has been reported previously that the promoter of many eye-specific genes, including all four Drosophila rh genes, contains a very conserved sequence (RCS1). This sequence has been shown to be critical for Drosophila rh1 as well as for $r$ h3 and rh4 gene expression (Mismer and Rubin 1987; Fortini and Rubin 1990). We have also defined this sequence, called P3, as the optimal binding site for Prdtype HD dimers (Wilson et al. 1995). Because Pax-6 has been proposed to be a regulator of $r h$ gene expression (Zuker 1994), and because it encodes a protein with a Paired-type HD (whose optimal binding site has been defined as P3; Czerny and Busslinger 1995), this raised the possibility that Pax- 6 may act through these sites to control photoreceptor-specific gene expression. Recently we have cloned a new R8-specific rh gene, rh5, whose proximal promoter also contains this conserved $\mathrm{P} 3$ sequence (Papatsenko et al. 1997). In the adult fly, $r h 1$ is expressed specifically in photoreceptor cells R1 to R6, and this expression can be recapitulated by a short promoter fragment $(-252$ to +67$)$ attached to lacZ (Mismer and Rubin 1987). To test whether ey is involved in the regulation of the $r h 1$ gene through binding to this conserved P3 site, we performed change-of-specificity experiments as follows.

We first tested the ability of Pax-6 subdomains
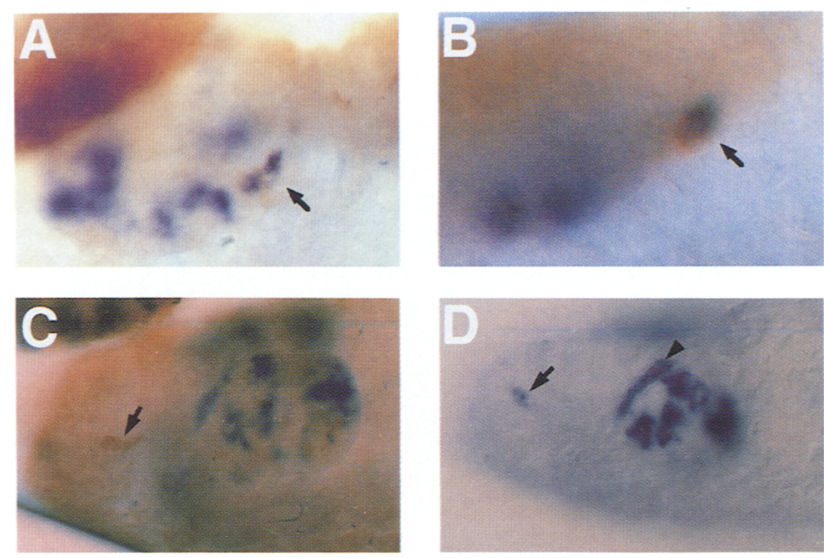

Figure 2. ey expression in the $\mathrm{BO}$ precursor cells. $\{A, B)$ In situ double labelings of a stage 12 embryo with ey (blue) and $K r$ (brown) probes. $K r$ can be detected in four to six BO precursor cells. (A) Three progenitor cells expressing $K r$ (magnification, 66×). Among these three cells, two also express ey (arrow). (B) Single cell (arrow) expressing both $K r$ (brown) and ey (blue) (magnification, 100x). (C) Early stage 16 embryo double-labeled with ey (blue) and $K r$ (brown) probes. $K r$ is clearly expressed in 12 BO cells (arrow), which have migrated to the anterior head region. ey is not expressed in these cells. (D) Late stage $16 \mathrm{em}-$ bryo labeled with ey (blue) probe. BO cells now clearly express ey (arrow). ey expression can also be seen in the optic lobe, many parts of the central brain, and the primordium of eye disc (arrowhead).

(Paired-type HD and PD) to bind in vitro to this minimal promoter and to several mutant promoters. Details of these promoters are shown in Figure 3A. Gel-shift assays showed that the Pax-6 HD recognizes the Drh-P3 fragment (the minimal $r$ promoter with a consensus $\mathrm{P} 3$ site) as a dimer (Fig. 3B), whereas, as expected, the Pax-6 PD did not bind to Drh-P3 (Fig. 3F). Becuase mutation of an 11-bp sequence overlapping the P3 site leads to loss of reporter gene expression in head extracts (Mismer and Rubin 1989|, we created Drh-P3Mut, in which the P3 site was mutated. This promoter fragment was not bound by either the Pax-6 HD (Fig. 3C) or PD (Fig. 3G). This shows that the $\mathrm{P} 3$ site in the minimal rh1 promoter is the only HD dimeric binding site, and that there is no detectable PD binding site on this promoter.

Although ey encodes a protein with both a PD and an $\mathrm{HD}$, it appears to recognize Drh-P3 only through its HD. We reasoned that it may also be able to act on the rh1 promoter through its PD if the P3 site was replaced by PD sites, even though the PD recognition sequence bears no resemblance to the P3 site. We thus tested Drh-PDS, which has two Pax-6 PD-binding sites, in opposite orientations, flanking both sides of the mutated P3 site. This site has been defined as optimal for Pax-6 PD, and is significantly less well recognized by other Pax molecules (Epstein et al. 1994a; Jun and Desplan 1996). We placed the Pax-6 PD sites in reverse orientation flanking the mutated P3 site. The HD was unable to bind to Drh-PDS (Fig. 3D), but the PD could now bind, first as one monomer, and then as two noncooperative monomers (Fig. 
Figure 3. Gel-shift assays with the Pax-6 $\mathrm{HD}$ and PD. (A) Wild-type and mutant promoters tested in gel-shift and transgenic assays. Drh-P3 has a consensus pairedtype HD-binding site P3 (TAATTGAATTA) located from -65 bp to $-75 \mathrm{bp}$ in the promoter. In Drh-P3Mut, the P3 site has been replaced by GCGAC TAGTCC. Drh-PDS has a Pax-6 PD-binding site (TTCACGCTTGACTGCATC. GAGG) on both sides of P3Mut. DrhP3 + PDS adds the Pax-6 PD-binding site on both sides of P3. The PD and HD sites are in opposite orientation, which allows the cooperative DNA binding by the PD and the HD. $(B-E)^{32} \mathrm{P}$-labeled Drh-P3, Drh-P3Mut, Drh-PDS, and Drh-P3 + PDS promoters shifted with $3 \times$ serially diluted amounts of Pax- $6 \mathrm{HD}$. The HD bound preferably to $\operatorname{DrhP} 3(B)$ and DrhP3 + PDS $(E)$ as a dimer, but did not bind to Drh-P3Mut $(C)$ or Drh-PDS $(D)$. The lower shift band seen in $D$ and $E$ is attributable to nonspecific binding. $(F-I){ }^{32}$ P-labeled Drh-P3, Drh-P3Mut, Drh-PDS, and Drh-P3 + PDS promoters, respectively, shifted with $3 \times$ serially diluted amounts of the Pax-6 PD. The Pax-6 PD was able to bind (first as one monomer and then as two monomers) to the Drh-PDS $(H)$ and the Drh-P3 + PDS $(I)$, which have two PD sites. Neither the Drh-P3 $(F)$ nor the Drh-P3Mut $(G)$ were bound by the $\mathrm{PD}$, indicating that no PD site is present in the minimal promoter.

$3 \mathrm{H})$. We also tested the promoter Drh-P3 + PDS, in which both the $\mathrm{P} 3$ and the two PD sites are present. This configuration allows both cooperative binding between the HD and the PD within each Pax- 6 molecule, as well as dimerization of two Pax-6 molecules through their HD. Our previous molecular and structural modeling studies predict that this configuration would be the highest affinity site for two Pax-6 molecules binding through a HD-induced dimer (Jun and Desplan 1996). Both the HD and the PD are now able to bind to the promoter Drh-P3 + PDS (Fig. 3E,I).

\section{Pax- 6 regulates in vivo rhl expression by binding to the P3/RCS1 site}

To test whether Pax- 6 is able to bind to the P3/RCS1 site of the rh1 promoter and regulate its expression, we generated transgenic lines from four types of constructs. Each construct contained the $1 a c Z$ reporter gene driven by $r h 1$ promoter with either a $\mathrm{P} 3$ site (Drh-P3) or various mutated sites (Drh-P3Mut, Drh-PDS, and DrhP3 + PDS) as described above (Fig. 3A). Adult heads from homozygous transgenic lines of each construct were sectioned for $\beta$-galactosidase activity assays. Drh-P3 was able to drive strong and highly specific lac $Z$ expression in outer photoreceptor cells R1 to R6 (Fig. 4A), where rh1 is normally expressed (Mismer and Rubin 1987). When we mutated the P3 site (Drh-P3Mut), the $r h 1$ promoter could no longer drive lac $Z$ expression in R1-R6 photoreceptor cells of the compound eyes (Fig. 4B), and the signal was not detected in any other photoreceptor cells in the adult $(R 7, R 8$, and ocelli). This confirmed that the P3 site is critical for the regulation of rh1 gene expression (Mismer and Rubin 1987). When, in addition to abolishing the P3 site, we added in the flanking sequences two Pax-6-specific PD-binding sites (Drh-PDS), the promoter regained its ability to direct specific and strong expression in the R1-R6 cells (Fig. 4C). This argues that the factor normally acting on the P3 site contains both a Paired-type HD and a Pax-6-like PD. Further support for this conclusion came from the fact that, when both the PD and HD sites were present in a synergistic combination (Drh-P3 + PDS), the promoter responded much more strongly than the promoter with either the P3 HD site alone (Drh-P3) or the PD sites alone (Drh-PDS). Strong lacZ expression was detected in R1-R6, and weaker expression became detectable in R7 and R8 as indicated by the staining, not only of the lamina of the optic lobe where R1-R6 project, but also of the medulla where R7 and R8 project (Fig. 4D). When Pax-6 PD and HD sites are associated in the predicted synergistic combination, as in Drh-P3 + PDS, these very high affinity Pax- 6 binding sites may allow the rh1 promoter to direct expression not only in the R1-R6 photoreceptor cells where it is normally expressed, but also in photoreceptors that normally express a different type of opsin gene ( $r h 3$ and $r h 4$ are expressed in $\mathrm{R} 7$ and at least rh5 is expressed in R8) (Fortini and Rubin 1990; Papatsenko et al. 1997). Considering that the P3 and PD sites are specific for Pax-6, these results strongly suggest that Drosophila Pax-6, or a very close homolog of Pax-6, is the molecule that binds to the P3 site in the rh1 pro- 


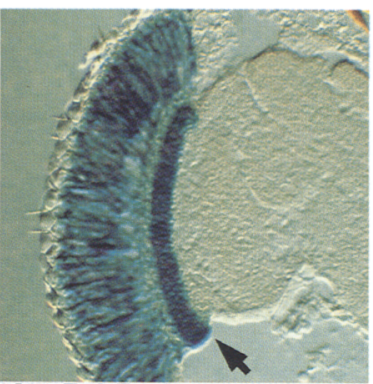

A DrhP3-lacZ
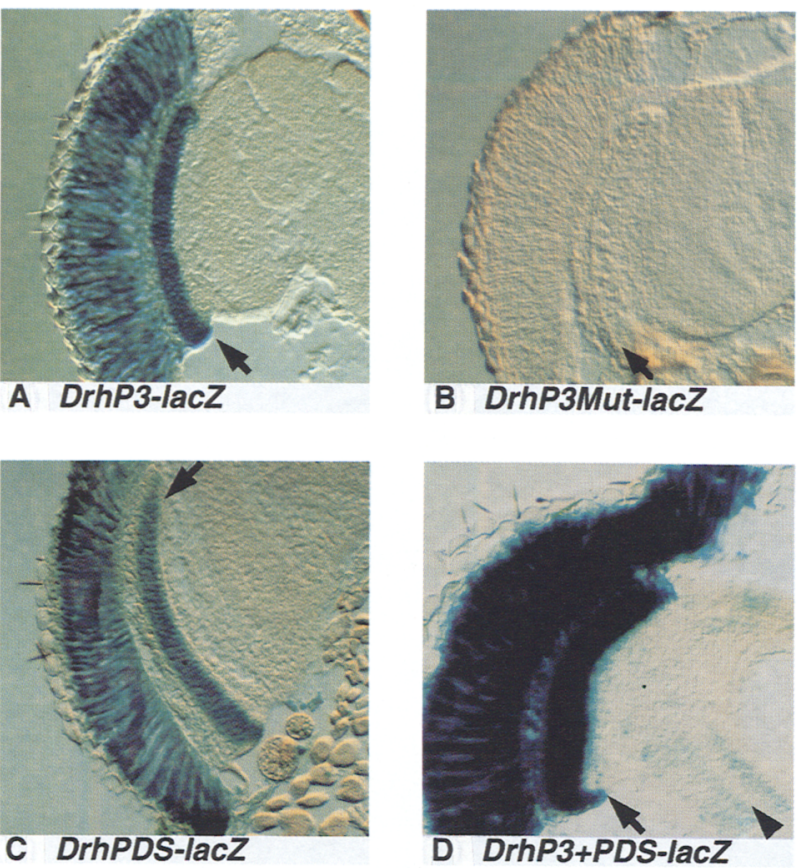

Figure 4. Lac $Z$ activity stainings of head sections with transgenic fly lines carrying DrhP3-lacZ $(A)$, DrhP3Mut-lacZ $(B)$, DrhPDS-lacZ $|C|$, and DrhP3 + PDS-lacZ $(D)$. The lamina is indicated by the arrow and the medulla by the arrowhead. $|A|$ Strong staining in the compound eyes was detected for DrhP3lacZ. Only photoreceptor cells R1-R6 showed the staining, because only projections to the lamina were detected. $(B)$ Mutation in the P3 site totally abolished the photoreceptor-specific staining. No specific staining was detected in the head sections with the DrhP3Mut-IacZ lines. $(C)$ Addition of the PD binding sites rescued the promoter activity, and the R1-R6 photoreceptor cells regained lacZ expression at normal level. $(D)$ When both the HD-binding sites (P3) and the PD-binding sites (Pax-6 PDS| were present, the promoter became much stronger and less restricted to the R1-R6 cells. The R1-R6 photoreceptors expressed lac $Z$ at much higher level (arrow), whereas the R7 and R8 cells started to express lac $Z$ as shown by staining of the medulla (arrowhead).

moter and regulates the expression of the rh1 gene in the adult fly.

\section{$P 3 / R C S 1$ site is a general element in photoreceptor-specific promoters}

The P3/RCS1 sequence is conserved in all known insect $r h$ genes as well as in many other terminal photoreceptor-specific genes (Wilson et al. 1995). This suggests that many terminal photoreceptor-specific genes may be regulated by Pax-6. This is consistent with the hypothesis of an ancestral role for Pax-6 in primitive photoreceptors where it regulates terminal photoreceptor-specific gene expression. We then asked whether this conserved P3 site could be sufficient for regulating photoreceptor cell-specific gene expression in Drosophila. We constructed a P-element vector with the lac $Z$ reporter gene driven by multimerized P3 sites up- stream of a basal promoter. Transgenic lines containing this construct exhibited strong lacZ expression in the adult eyes (Fig. 5). All photoreceptor cells in the compound eye (R1-R8) expressed lacZ, as shown by projections of photoreceptor axons to both the lamina and the medulla (proximal and distal) regions of the optic lobe (Fig. 5A). This spectrum is wider than that of any $r h$ gene expressed in the compound eyes. Interestingly, lac $Z$ was also expressed in the other adult visual structures, the ocelli (Fig. 5B), where yet another opsin gene, rh2, is expressed (Pollock and Benzer 1988). lacZ expression was also detected in the larval visual system (Bolwig organs; data not shown), suggesting that the same regulator is active in all photoreceptor cells and ey regulates gene expression in different types of photoreceptor cells through binding to the conserved P3 sequence. Because all Drosophila th genes have the conserved P3/RCS1 sequence in their promoters, this suggests that P3 site promotes expression in all photoreceptor cells. In all four Drosophila rh promoters that have been analyzed so far, a subtype-specific promoter sequence is always present together with the general P3/RCS1 sequence (Fortini and Rubin 1990). Neither RCS1 nor the subtype-specific element is sufficient by itself to drive the $r h$ gene expression. The optimized and multimerized nature of P3 sequences in the promoter construct may have overcome the combinatorial requirement for subtype-specific factors in subsets of the photoreceptor cell types. In this case, however, the subtype-specific $r h$ expression was also lost and all photoreceptor cells express the reporter gene.
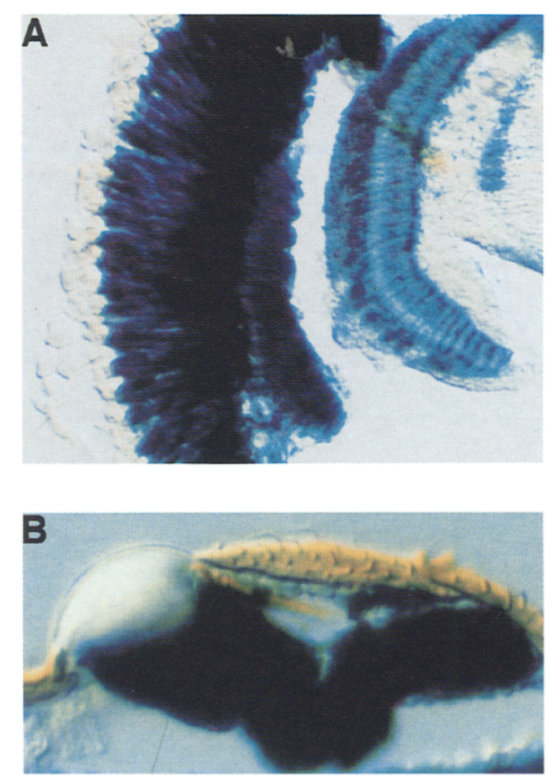

Figure 5. Expression of the lacZ reporter gene driven by multiple P3 sites. (A) Both outer (R1-R6) and inner (R7 and R8) photoreceptor cells in the compound eyes strongly expressed lac $Z$. This expression level was much higher than with DrhP3 + PDS-lacZ lines. (B) Strong staining is also detected in ocelli. The photoreceptor cells in the ocelli express $r h 2$, whose promoter also contains a $\mathrm{P} 3$ site. 


\section{Discussion}

ey is required in both early and late stages of eye development

In the early stages of eye development, ey is expressed in the developing eye discs and the proliferating precursor cells of the larval photoreceptors. Its function in early stages may be to maintain the identity of photoreceptor progenitor cells and also to induce the proliferation of these cells. ey expression disappears after the initial differentiation of photoreceptor cells (Quiring et al. 1994). We showed that ey expression is reactivated later in the photoreceptor cells that have started terminal differentiation. Its function during this stage may be to regulate the expression of structural genes like $r h$. This latter function may be more ancient because terminal photoreceptor-specific genes like $r h$ are among the few evolutionarily conserved features of different metazoan photoreceptive organs.

If the regulation of the terminal photoreceptor-specific gene expression is the ancestral role of Pax-6/ey, one would expect to find this expression to be conserved in all photoreceptive systems. This appears to be true in Drosophila where ey likely regulates terminal photoreceptor-specific genes (e.g., $r h$ ) in all three visual systems (the compound eyes, the ocelli, and the $\mathrm{BO}$ ). In vertebrates, reports have described Pax- 6 expression in the differentiating neuroretina in mouse (Ton et al. 1991; Walther and Gruss 1991), zebrafish (Puschel et al. 1992), and quail (Carriere et al. 1993) but not in the differentiated photoreceptors. However, more recent reports (Korona et al. 1997; R. Maas, pers. comm.) have unambiguously identified Pax-6 expression in photoreceptor cells after the last mitotic division of photoreceptor progenitor cells, and this expression remains at least until birth. Although one report indicates that Pax- 6 is not expressed in the adult photoreceptor cells of chick, mouse, and monkey (Korona et al. 1997), it is possible that the regulation of terminal photoreceptor-specific genes in these organisms occurs at the postmitotic and preadult stages. The postmitotic expression of Pax-6 in mouse supports a role in regulating opsin gene expression in vertebrates. The absence of $P a x-6$ expression or the undetected low-level expression in adult photoreceptor cells in some vertebrate organisms could indicate a possible role of Pax- 6 in opening the chromatin structure to allow transcription initiation. Pax- 6 may not be required for the maintenance of this expression. Pax- 6 appears to be expressed in adult lens epithelium of many vertebrates and to regulate the expression of another type of terminal eye-specific gene, the crystallins (Cvekl et al. 1995a,b; Richardson et al. 1995; Cvekl and Piatigorsky 1996). However, this regulation appears to involve the PD of Pax-6, not the HD as is the case for the $r h$ gene regulation.

\section{A distinct form of ey transcript is expressed in the adult stage}

As ey is required in multiple developmental processes (Quiring et al. 1994; Quinn et al. 1996) including differ- ent stages of eye development, it was not surprising to find a novel adult-specific form of $e y$ transcript. The fact that it contains both the E10 (embryonic-specific) first exon and the D1 (larval-specific) second exon suggests that the adult-specific form has a different transcriptional start from the larval-specific form and is likely to be controlled by a distinct regulatory mechanism. This is consistent with our observation that $e y$ is turned off after the initial determination of photoreceptor cells and is turned on only later, by a different control mechanism. It is not clear whether the adult-specific form and the embryonic-specific E10 form share the same promoter and are only splice variants. From a molecular point of view, all three forms vary only in their very amino-terminal region and have intact $\mathrm{PD}$ and $\mathrm{HD}$. It will be interesting to know whether these differences among the three forms can cause functional differences in transcriptional activities of the Ey protein. From studies of Pax-6 homologs in other species, it has been shown that alternative splicing (Epstein et al. 1994b) and post-translational modification (Carriere et al. 1993) of Pax-6 can generate functional diversity. This diversity includes isoforms of Pax-6 that lack one of the functional domains (the PD and the HD|. In Caenorhabditis elegans, two separate genetic functions $(v a b-3$ and $m a b-18)$ are controlled by two forms of Pax-6, one with both the PD and the HD $(v a b-3)$ and the other with only the HD (mab-18) (Chisholm and Horvitz 1995; Zhang and Emmons 1995).

\section{The HD of ey binds to P3/RCS1 element in the rhodopsin promoters}

We demonstrated that the HD of Pax-6/Ey is able to bind to the P3/RCS1 element. The fact that binding sites specific for the PD of Pax-6/Ey class are able to replace the function of P3/RCS1 in vivo suggests that this element is bound by a protein with both a Pax-6 class PD and a Paired-class HD, or, alternatively, by two proteins each containing a Pax- 6 class PD or a Paired-class HD. The second option is unlikely because all proteins with a Pax-6 class PD contain a Paired-class HD. Further support for the presence in a single molecule of both a Pax-6 class PD and a Paired-class HD comes from the fact that composite sites for both the HD and PD mediate synergistically increased activity of the promoter, which is likely to reflect the intramolecular cooperativity between the PD and the HD in vitro (Jun and Desplan 1996). Together, this strongly suggests that Ey binds to the P3/RCS1 element in $r h$ promoters. Although we cannot rule out the possibility that a close homolog of ey (e.g., twin of eyeless; Callaerts et al. 1997) acts on these sites, we can conclude that a Drosophila Pax-6 homolog is the transcription factor that binds to the P3/RCS1 element and regulates the expression of $r h$ genes.

The P3/RCS1 element is a general cis-regulatory element for terminal photoreceptor-specific gene expression

The P3/RCS1 element was first defined as an essential conserved element in all known Drosophila rh promot- 
ers (Mismer and Rubin 1989; Fortini and Rubin 1990). The essential function of this element was further confirmed by the presence of this element in a similar proximal region of the promoter of a newly discovered R8specific rhodopsin (rh5) (Papatsenko et al. 1997). This element was also found in the promoter of many of the photoreceptor-specific genes (Mismer and Rubin 1989; Wilson et al. 1995) and was reported to be essential for the expression of rat opsin (Yu and Barnstable 1994-95), suggesting that the P3/RCS1 sequence functions as a general photoreceptor-specific cis-regulatory element. It has been reported that there is at least another general photoreceptor-specific cis-regulatory element, a 27-bp element in rh1 promoter (Moses and Rubin 1991; Sheshberadaran and Takahashi 1994). This element (from -194 to -220 ) was identified as a glass protein-binding site (Moses et al. 1989; Moses and Rubin 1991; Ellis et al. 1993; Sheshberadaran and Takahashi 1994), and multiple copies of this element can drive a reporter gene expression in all photoreceptor cells (Moses and Rubin 1991; Ellis et al. 1993). In this report, we showed that multimerized P3/RCS1 elements are able to drive the expression of a reporter gene in all photoreceptor cells, without discrimination for subtypes. This suggests two important features in the regulation of photoreceptor cell-specific genes. One is that the P3/RCS1 element is normally insufficient to activate gene expression and requires cofactors that recognize other elements, including general enhancer elements like the 27-bp glass binding site and (photoreceptor subtype-specific elements (Mismer and Rubin 1989; Fortini and Rubin 1990) in the promoter for activation. The second important feature is that the subtype-specific cis-regulatory elements are essential to provide specificity for photoreceptor gene expression. For complex photoreceptive organs like the Drosophila compound eye and the vertebrate eye, generating different subtypes of photoreceptor cells is essential for their functions (e.g., color vision and polarized light detection). Promoter analyses of the Drosophila rh genes have supported this (Mismer and Rubin 1989; Fortini and Rubin 1990; A. Tahayato and C. Desplan, unpubl.). The requirement for subtype-specific elements, however, can be bypassed by multimerizing the P3/ RCS1 site.

The regulation of $\mathrm{rhl}$ by Pax-6/ey supports the model of monophyletic evolution of metazoan photoreceptive organs

Besides Drosophila and vertebrates, Pax-6 has also been found in flatworm, ribbon worm, nematode, squid, sea urchin, and ascidian (Callaerts et al. 1997). These organisms represent metazoan phyla with distinct eye structures, and sometimes with no eye structure. $P a x-6$ is always expressed in the developing eyes (in organisms that have eyes) and the central nervous system. The genetic and molecular study of Drosophila ey indicates that $\operatorname{Pax}-6$ is likely to be at the top of the regulatory hierarchy specific for eye development. Two models have been proposed to explain the presence of Pax-6 in divergent eyes. One model argues against the common origin of metazoan eyes and proposes that the involvement of Pax-6 in different types of eyes can be explained by independent recruitment of Pax- 6 after the eyes have diversified (Nilsson 1996). A more parsimonious explanation invokes a common origin for the function of this regulator in both insect and vertebrate eyes, and by extension perhaps those of all metazoans (Zuker 1994; Halder et al. 1995b). Reconciling the recent molecular data with classical phylogenetical studies, this model suggests that Pax-6 is locked in the regulatory pathway of eye development because of its more ancient function in the direct regulation of terminal photoreception genes like $r h$ (Zuker 1994). Later in evolution, genes specific for each type of eye may have been added to this regulatory pathway to specify divergent and complex eye structures. The evidence we have shown here for the direct regulation of $r h 1$ genes by Pax- 6 suggests that the primitive function of Pax-6 is to regulate structural genes required for elementary photoreception, and that this function has been retained in complex eyes such as those of Drosophila.

\section{Materials and methods}

\section{RT-PCR}

Total RNA was extracted using TRI Reagent (Molecular Research Center, Inc., Ohio). Approximately $10 \mu \mathrm{g}$ of total RNA was used for reverse transcription with 10 pmoles of specific primers. The reaction was carried out in $50 \mathrm{~mm}$ Tris- $\mathrm{HCl} / \mathrm{pH}$ 8.3), $75 \mathrm{~mm} \mathrm{KCl}, 3 \mathrm{~mm} \mathrm{MgCl}_{2}, 10 \mathrm{~mm}$ dNTPs, $10 \mathrm{~mm} \mathrm{DTT,} 1$ $\mathrm{U} / \mu \mathrm{l}$ of RNase inhibitor (Promega), and $4 \mathrm{U} / \mu \mathrm{l}$ of $\mathrm{M}-\mathrm{MuLV}$ reverse transcriptase (Stratagene) in $20 \mu \mathrm{l}$ final volume for $1 \mathrm{hr}$ at $37^{\circ} \mathrm{C}$. PCR was carried out as $25-35$ cycles of $94^{\circ} \mathrm{C}$ for $2 \mathrm{~min}$, $50^{\circ} \mathrm{C}$ for $2 \mathrm{~min}$ and $72^{\circ} \mathrm{C}$ for $40 \mathrm{sec}$. Primers used for the PCR reactions were as follows. ey HD-specific primers: $5^{\prime}$-end primer, 5'-GAGGATGATCAAGCTCGGCTCATAC-3' and 3'end primer, 5' -TTTCGCAGCTTCTCCTCGCGACGCC-3' . ey PD-specific primers: 5'-end primer, 5'-GGGTCACAGTGGAGTAAATCAGCTG-3' and $3^{\prime}$-end primer, 5'-AACGTGCCTCTCGATCCGC-3'. Rp49-specific primers: $5^{\prime}$-end primer, 5'CCAAGGACTTCATCCGCCACC- $3^{\prime}$ and $3^{\prime}$-end primer, $5^{\prime}$ GCGGGTGCGCTTGTTCGATCC- ${ }^{\prime}$. All $r h$-specific primers: $5^{\prime}$-end primer, degenerative oligonucleotide corresponding to conserved QAKK. 3'-end primer, degenerative oligonucleotide corresponding to conserved WTPY. rh1-specific primers: $5^{\prime}$-end primer, 5'-TTCCAACGACCAATCGC-3' and $3^{\prime}$ 'end primer, 5'-AGGGTCCTGGATTCTGATA-3'.

\section{In situ staining of Drosophila embryos}

Overnight collection of wild-type Drosophila embryos (0-18 hr) were dechorionated in $50 \%$ bleach and fixed in $10 \%$ paraformaldehyde for $20 \mathrm{~min}$. Digoxigenin-labeled $\mathrm{Kr}$ probe and fluorescein-labeled ey probe (digoxigenin or fluorescein labeling mix, Boehringer-Mannheim) were used for hybridization. The hybridization was carried out in $50 \%$ formamide, $5 \times$ SSC, 200 $\mu \mathrm{g} / \mathrm{ml}$ of sonicated salmon sperm DNA, $100 \mu \mathrm{g} / \mathrm{ml}$ of tRNA, and $50 \mu \mathrm{g} / \mathrm{ml}$ of heparin adjusted to $\mathrm{pH} 5.0$ overnight at $70^{\circ} \mathrm{C}$. After several washing steps (first in hybridization solution, then in PBS-Tween20), embryos were incubated with anti-digoxigenin antibody coupled with peroxidase and anti-fluorescein an- 
tibody coupled with alkaline phosphatase (both from Boehringer Mannheim) for $1 \mathrm{hr}$.

\section{Gel mobility shift assay}

The ey $\mathrm{HD}$, including sequences encoding 4 amino acid residues upstream and 10 amino acid residues downstream, was cloned in pET-14b vector. ey HD expressed in BL21(DE3) bacteria was used for gel shift. Protein-DNA binding was carried out in 20 $\mathrm{mm}$ Tris (pH 7.5), 10\% glycerol, $50 \mathrm{~mm} \mathrm{KCl,} 1 \mathrm{~mm}$ DTT, 0.5 $\mathrm{mg} / \mathrm{ml}$ of BSA, $100 \mu \mathrm{g} / \mathrm{ml}$ of herring sperm DNA, and $100 \mu \mathrm{g} /$ $\mathrm{ml}$ of poly[d(I-C)] for $20 \mathrm{~min}$. Purified mouse Pax-6 PD was used for the gel shift. Mouse Pax-6 PD is $96 \%$ identical to the ey PD and mouse $\mathrm{Pax}-6$ has the same potential as ey in inducing ectopic eyes in vivo. The reaction was carried out with the same condition as in the HD-DNA binding.

\section{Generation of transgenic lines and X-gal stainings on adult head sections}

Nine DrhP3-lacZ lines, six DrhP3Mut-lacZ lines, eight DrhP$D S-l a c Z$ lines, and six DrhP3 + PDS-lacZ lines were generated. Homozygous fly heads were fixed and cryo-sectioned before staining. X-gal staining was performed in $1 \times \mathrm{PBS}, 2 \mathrm{~mm} \mathrm{MgCl}_{2}$, $5 \mathrm{mM} \mathrm{K}_{3} \mathrm{Fe}(\mathrm{CN})_{6}, 5 \mathrm{mM} \mathrm{K}_{4} \mathrm{Fe}(\mathrm{CN})_{6}$ and $1 \mathrm{mg} / \mathrm{ml} \mathrm{X}$-gal for $10 \mathrm{~min}$ to $1 \mathrm{hr}$. All lines with the same construct gave similar staining pattern and staining intensity. For generating flies carrying P3 sites driving $l a c Z$, nine copies of the P3 site were cloned upstream of the basal heat-shock promoter driving lacZ. Ten lines were generated and assayed for $\beta$-galactosidase activity. X-gal staining was carried out in the same condition as above.

\section{Acknowledgments}

We thank Patrick Callaerts and Walter Gehring for the ey cDNA and Richard Maas for the purified Pax-6 PD. We are indebted to Ulrike Gaul, Susie Jun, and Jessica Treisman for many discussions and insightful suggestions, and Chizi Gong for the help with the staining on head sections. We thank also all other members of the Desplan laboratory for many helpful discussions.

The publication costs of this article were defrayed in part by payment of page charges. This article must therefore be hereby marked "advertisement" in accordance with 18 USC section 1734 solely to indicate this fact.

\section{References}

Bolwig, N. 1946. Senses and sense organs of the anterior end of the house fly larva. Vidensk. Medd. Dan. Naturhist. Foren. 109: 80-212.

Callaerts, P., G. Halder, and W.J. Gehring. 1997. Pax-6 in development and evolution. Annu. Rev. Neurosci. 20: (in press).

Carriere, C., S. Plaza, P. Martin, B. Quatannens, M. Bailly, D. Stehelin, and S. Saule. 1993. Characterization of quail Pax-6 (Pax-QNR) proteins expressed in the neuroretina. Mol. Cell. Biol. 13: 7257-7266.

Chalepakis, G. and P. Gruss. 1995. Identification of DNA recognition sequences for the Pax3 paired domain. Gene 162: $267-270$.

Cheyette, B., P. Green, K. Martin, H. Garren, V. Hartenstein, and S. Zipursky. 1994. The Drosophila sine oculis locus encodes a homeodomain-containing protein required for the development of the entire visual system. Neuron 12: $997-$ 996.
Chisholm, A. and H. Horvitz. 1995. Patterning of the Caenorhabditis elegans head region by the Pax- 6 family member vab-3. Nature 376: 52-55.

Cvekl, A. and J. Piatigorsky. 1996. Lens development and crystallin gene expression: Many roles for Pax-6. BioEssays 18: $621-630$.

Cvekl, A., C. Sax, X. Li, J. McDermott, and J. Piatigorsky. 1995a. Pax- 6 and lens-specific transcription of the chicken delta 1-crystallin gene. Proc. Natl. Acad. Sci. 92:4681-4685.

Cvekl, A., F. Kashanchi, C. Sax, J. Brady, and J. Piatigorsky. 1995b. Transcriptional regulation of the mouse alpha A-crystallin gene: Activation dependent on a cyclic AMPresponsive element (DE1/CRE) and a Pax-6-binding site. Mol. Cell. Biol. 15: 653-660.

Czerny, T. and M. Busslinger. 1995. DNA-binding and transactivation properties of Pax-6: Three amino acids in the paired domain are responsible for the different sequence recognition of Pax-6 and BSAP (Pax-5). Mol. Cell. Biol. 15: 28582871.

Czerny, T., G. Schaffner, and M. Busslinger. 1993. DNA sequence recognition by Pax proteins: Bipartite structure of the paired domain and its binding site. Genes \& Dev. 7: 20482061.

Ellis, M., E. O'Neill, and G. Rubin. 1993. Expression of Drosophila glass protein and evidence for negative regulation of its acitivity in non-neuronal cells by another DNA-binding protein. Development 119: 855-865.

Epstein J., J. Cai, T. Glaser, L. Jepeal, and R. Maas. 1994a. Identification of a Pax paired domain recognition sequence and evidence for DNA-dependent conformational changes. J. Biol. Chem. 269: 8355-8361.

Epstein, J., T. Glaser, J. Cai, L. Jepeal, D. Walton, and R. Maas. 1994b. Two independent and interactive DNA-binding subdomains of the Pax6 paired domain are regulated by alternative splicing. Genes \& Dev. 8: 2022-2034.

Fortini, M. and G. Rubin. 1990. Analysis of cis-acting requirements of the Rh3 and Rh4 genes reveals a bipartite organization to rhodopsin promoters in Drosophila melanogaster. Genes \& Dev. 4: 444-463.

Green, P., A. Hartenstein, and V. Hartenstein. 1993. The embryonic development of the Drosophila visual system. Cell Tissue Res. 273: 583-598.

Gruss, P. and C. Walther. 1992. Pax in development. Cell 69: 719-722.

Halder, G., P. Callaerts, and W. Gehring. 1995a. Induction of ectopic eyes by targeted expression of the eyeless gene in Drosophila. Science 267: 1788-1792.

1995b. New Perspectives on eye evolution. Curr. Opin. Genet. Dev. 5: 602-609.

Hill, R., J. Favor, B. Hogan, C. Ton, G. Saunders, I. Hanson, J. Prosser, T. Jordan, N. Hastie, and V. van Heyningen. 1991. Mouse small eye results from mutations in a paired-like homeobox-containing gene. Nature 354: 522-525.

Jun, S. and C. Desplan. 1996. Cooperative interaction between homedomain and paired domain. Development 122: 26392650.

Korona, B., J.M. Yang, and O.H. Sundin. 1997. The Pax-6 homeobox gene is expressed throughout the corneal and conjunctival epithelia. Invest. Ophthalmol. Visual Sci. 38: 108120.

Kumar, J. and D. Ready. 1995. Rhodopsin plays an essential structural role in Drosophila photoreceptor development. Development 121: 4359-4370.

Mismer, D. and G. Rubin. 1987. Analysis of the promoter of the ninaE opsin gene in Drosophila melanogaster. Genetics 116: $565-578$. 
1989. Definition of cis-acting elements regulating expression of the Drosophila melanogaster ninaE opsin gene by oligonucleotide-directed mutagenesis. Genetics 121: 77-87.

Moses, K. and G. Rubin. 1991. Glass encodes a site-specific DNA-binding protein that is regulated in response to positional signals in the developing Drosophila eye. Genes \& Dev. 5: 583-593.

Moses, K., M. Ellis, and G. Rubin. 1989. The glass gene encodes a zinc-finger protein required by Drosophila photoreceptor cells. Nature 340: 531-536.

Nilsson, D.E. 1996. Eye ancestry: Old genes for new eyes. Curr. Biol. 6: 39-42.

Oliver, G., A. Mailhos, R. Wehr, N. Copeland, N. Jenkins, and P. Gruss. 1995. Six3, a murine homologue of the sine oculis gene, demarcates the most anterior border of the developing neural plate and is expressed during eye development. Development 121: 4045-4055.

Papatsenko, D., G. Sheng, and C. Desplan. 1997. A new Rhodopsin in R8 photoreceptors of Drosophila and evidence for coordinate expression with Rh3 in R7 cells. Development 124: $1665-1673$.

Plaza, S., C. Dozier, N. Turque, and S. Saule. 1995. Quail Pax-6 (Pax-QNR) mRNAs are expressed from two promoters used differentially during retina development and neuronal differentiation. Mol. Cell. Biol. 15: 3344-3353.

Pollock, J. and S. Benzer. 1988. Transcript localization of four opsin genes in the three visual organs of Drosophila; RH2 is ocellus specific. Nature 333: 779-782.

Puschel, A., P. Gruss, and M. Westerfield. 1992. Sequence and expression pattern of pax-6 are highly conserved between zebrafish and mice. Development 114: 643-651.

Quinn, J., J. West, and R. Hill. 1996. Multiple functions for Pax6 in mouse eye and nasal development. Genes \& Dev. 10: 435446.

Quiring, R., U. Walldorf, U. Kloter, and W.J. Gehring. 1994. Homology of the eyeless gene of Drosophila to the Small eye gene in mice and Aniridia in humans. Science 265: 785-789.

Richardson, J., A. Cvekl, and G. Wistow. 1995. Pax-6 is essential for lens-specific expression of zeta-crystallin. Proc. Natl. Acad. Sci. 92: 4676-4680.

Salvini-Plawen, L.V. and E. Mayr. 1977. On the evolution of photoreceptors and eyes. Evol. Biol. 10: 207-263.

Schmucker, D., H. Taubert, and H. Jäckle. 1992. Formation of the Drosophila larval photoreceptor organ and its neuronal differentiation require continuous Kruppel gene activity. Neuron 9: 1025-1039.

Schmucker, H., H. Jäckle, and U. Gaul. 1997. Genetic analysis of the larval optic nerve projection. Development 124: 934948.

Sheshberadaran, H. and J. Takahashi. 1994. Characterization of the chicken rhodopsin promoter: Identificiation of retinaspecific and glass-like protein binding domains. Mol. Cell. Neurosci. 5: 309-318.

Strachan, T. and A.P. Read. 1994. PAX genes. Curr. Opin. Genet. Dev. 4: 427-438.

Ton, C., H. Hirvonen, H. Miwa, M. Weil, P. Monaghan, T. Jordan, V. van Heyningen, N. Hastie, H. Meijers-Heijboer, M. Drechsler et al. 1991. Positional cloning and characterization of a paired box- and homeobox-containing gene from the aniridia region. Cell 67: 1059-1074.

Walther, C. and P. Gruss. 1991. Pax-6, a murine paired box gene, is expressed in the developing CNS. Development 113: 1435-1449.

Wilson, D., G. Sheng, T. Lecuit, N. Dostatni, and C. Desplan. 1993. Cooperative dimerization of paired class homeo domains on DNA. Genes \& Dev. 7: 2120-2134.
Wilson, D., B. Guenther, C. Desplan, and J. Kuriyan. 1995. High resolution crystal structure of a paired (Pax) clas cooperative homeodomain dimer on DNA. Cell 82: 709-719.

Wolff, T. and D.F. Ready. 1993. Pattern formation in the Drosphila retina. In The development of Drosophila melanogaster (ed. M. Bate and A. Martinez Arias), pp. 1277-1325. Cold Spring Harbor Laboratory Press, Cold Spring Harbor, NY.

Xu, W., M. Rould, S. Jun, C. Desplan, and C. Pabo. 1995. Crystal structure of a paired domain-DNA complex at $2.5 \AA$ resolution reveals structural basis for Pax developmental mutations. Cell 80: 639-650.

Yu, X. and C. Barnstable. 1994-95. Characterization and regulation of the protein binding to a cis-acting element, RET 1 , in the rat opsin promoter. J. Mol. Neurosci. 5: 259-271.

Zhang, Y. and S. Emmons. 1995. Specification of sense-organ identity by a Caenorhabditis elegans Pax- 6 homologue. $\mathrm{Na}$ ture 376: 55-59.

Zuker, C. 1994. On the evolution of eyes: Would you like it simple or compound? Science 265: 742-743. 


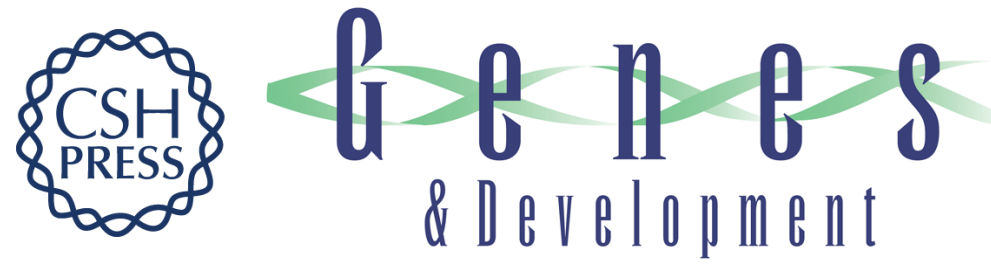

\section{Direct regulation of rhodopsin 1 by Pax-6/eyeless in Drosophila: evidence for a conserved function in photoreceptors.}

G Sheng, E Thouvenot, D Schmucker, et al.

Genes Dev. 1997, 11:

Access the most recent version at doi:10.1101/gad.11.9.1122

References This article cites 47 articles, 26 of which can be accessed free at:

http://genesdev.cshlp.org/content/11/9/1122.full.html\#ref-list-1

License

Email Alerting

Service

Receive free email alerts when new articles cite this article - sign up in the box at the top right corner of the article or click here.

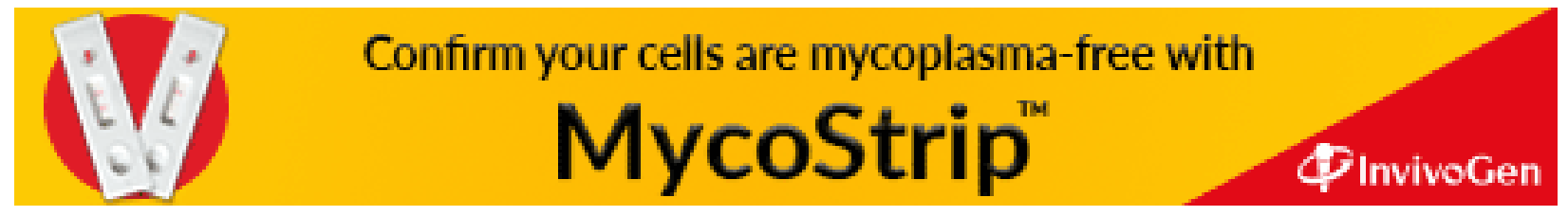

